\title{
Cancer-associated fibroblasts from invasive breast cancer have an attenuated capacity to secrete collagens
}

\author{
ZHIXUAN FU ${ }^{1,2^{*}}$, PEIMING SONG $^{1 *}$, DONGBO LI ${ }^{3}$, CHENGHAO YI $^{1}$, HUARONG CHEN $^{1}$, \\ SHUQIN RUAN ${ }^{1}$, ZHONG SHI $^{1}$, WENHONG XU ${ }^{1}$, XIANHUA FU $^{1}$ and SHU ZHENG ${ }^{1}$ \\ ${ }^{1}$ Key Laboratory of Cancer Prevention and Intervention (China National Ministry of Education), \\ The Second Affiliated Hospital, School of Medicine, Zhejiang University, Hangzhou, Zhejiang 310009; \\ ${ }^{2}$ Department of Surgical Oncology, Zhejiang Cancer Hospital, Hangzhou, Zhejiang 310022; \\ ${ }^{3}$ Cardiovascular Ward of Geriatric Department, The First Affiliated Hospital of \\ Zhengzhou University, Zhengzhou, Henan 450052, P.R. China
}

Received April 22, 2014; Accepted June 18, 2014

DOI: 10.3892/ijo.2014.2562

\begin{abstract}
Normal fibroblasts produce extracellular matrix (ECM) components that form the structural framework of tissues. Cancer-associated fibroblasts (CAFs) with an activated phenotype mainly contribute to ECM deposition and construction of cancer masses. However, the stroma of breast cancer tissues has been shown to be more complicated, and the mechanisms through which CAFs influence ECM deposition remain elusive. In this study, we found that the activated fibroblast marker $\alpha$-smooth muscle actin ( $\alpha$-SMA) was only present in the stroma of breast cancer tissue, and the CAFs isolated from invasive breast cancer sample remained to be activated and proliferative in passages. To further assess the difference between CAFs and normal breast fibroblasts (NFs), MALDI TOF/TOF-MS was used to analyze the secretory proteins of primary CAFs and NFs. In total, 2,903 and 3,023 proteins were identified. Mass spectrum quantitative assay and data analysis for extracellular proteins indicated that the CAFs produce less collagens and matrix-degrading enzymes compared with NFs. This finding was confirmed by western blot analysis. Furthermore, we discovered that reduced collagen deposition was present in the
\end{abstract}

Correspondence to: Professor Shu Zheng, Key Laboratory of Cancer Prevention and Intervention (China National Ministry of Education), The Second Affiliated Hospital, College of Medicine, Zhejiang University, Hangzhou, Zhejiang 310009, P.R. China

E-mail: zhengshu@zju.edu.cn

*Contributed equally

Abbreviations: CAFs, cancer-associated fibroblasts; NFs, normal bread fibroblasts; ECM, extracellular matrix; MAL-DI TOF/TOF-MS, matrix-assisted laser desorption/ionization time of flight mass spectrometry; MRM, multiple reaction monitoring; CPS, counts per second; SMA, smooth muscle actin; PDGFR, platelet derived growth factor receptor

Key words: cancer-associated fibroblasts, extracellular matrix, tumor microenvironment, mass spectrometry, desmoplasia stroma of invasive breast cancer. These studies showed that although CAFs from invasive breast cancer possess an activated phenotype, they secreted less collagen and induced less ECM deposition in cancer stroma. In cancer tissue, the remodeling of stromal structure and tumor microenvironment might, therefore, be attributed to the biological changes in CAFs including their protein expression profile.

\section{Introduction}

The main function of fibroblasts is secreting the components of extracellular matrix (ECM). Without activation, fibroblasts are dormant in the ECM, however, once activated, fibroblasts could be involved in secreting higher levels of ECM components and exert a stronger proliferative effect $(1,2)$. In the 1970s, Ryan et al originally described the activation of fibroblasts in granulation tissues during wound healing progression $(3,4)$. Activated fibroblasts are also referred to as myofibroblasts due to their expression of $\alpha$-smooth muscle actin ( $\alpha$-SMA), as well as other important functions $(5,6)$.

Carcinoma tissues are mainly composed of tumor cells and stromal cells, the latter include fibroblasts, endothelial and inflammatory cells. Fibroblasts, termed cancer-associated fibroblasts (CAFs) represent the most abundant cellular components in cancer stroma. The heterotypic and multicellular interactions among cells, soluble factors, signaling molecules, extracellular matrix construct a new biological system termed the 'tumor microenvironment (TME)'. Researchers have demonstrated that tumor cells and the TME coevolve through continuous paracrine communication (7), which not only creates a dynamic signaling circuitry that promotes cancer initiation and progress, but also induces the activation of fibroblasts (8). CAFs present in cancer stroma exhibit an activated phenotype analogous to that of fibroblasts involved in wound healing or fibrosis $(9,10)$. Indeed, Dvorak originally considered tumors to be 'wounds that do not heal', because of the similarity with granulation tissue (11). Increasing evidence supports the role of CAFs as a key regulator of the paracrine signaling required for cancer progression $(12,13)$. In contrast to resting fibroblasts, activated 
CAFs can be identified by their expression of vimentin, $\alpha$-SMA, fibroblasts activated protein (FAP), and platelet-derived growth factor receptor (PDGFR- $\beta$ ) (14-16). Kalluri has indicated that up to $80 \%$ of stromal fibroblasts in breast cancer display this activated phenotype (17). Furthermore, evidence indicates that CAFs neither revert back to normal fibroblasts nor undergo elimination via apoptosis (18).

CAFs are further characterized by their production of abundant ECM proteins, which are responsible for the stiffening appearance of cancer $(19,20)$. The deposition of ECM induced by CAFs in tumor stroma is known as desmoplasia, which has been shown to be extensive in or around tumors, particularly in human breast cancer and pancreatic carcinoma $(21,22)$. A study has shown that the compression of ECM in turn leads to the concentration of soluble factors that promote tumorigenesis in an autocrine and paracrine manner (13). However, Walker has reported that the stroma in a range of primary breast carcinomas could vary from being predominantly cellular with little collagens to being a dense collagenous stroma with apparently few stromal cells (23). These results are contradictory to many research results on the universal deposition of ECM in cancer tissue. Therefore, it is necessary to elucidate the relationship between activated CAFs and their function in ECM deposition. In this study, we aimed to investigate the secretomics of CAFs by mass spectrometry and other means in order to elucidate the relationship between CAFs and ECM deposition in breast cancer.

\section{Materials and methods}

Specimens. This study was based on a well-characterized series of TNM stage T2-T3 primary invasive breast carcinoma cases and fibroadenomas. Invasive breast cancer was selected for study due to its typical desmoplastic response and the presence of large numbers of activated CAFs. The fresh specimens of invasive carcinoma were collected from cancer patients who accepted radical mastectomy at the Second Affiliated Hospital of Zhejiang University. Written consent was obtained from all patients and this study was approved by the ethics committee of Zhejiang University. Tissue specimens used in immunohistochemistry and histochemistry were obtained from the tissue bank of the Cancer Institute, Zhejiang University.

Immunohistochemistry (IHC) and histochemistry. In total 160 cases of invasive breast cancer, 40 adjacent normal breast tissue and 6 fibroadenoma specimens were obtained. After the tissues were fixed with $4 \%$ paraformaldehyde and paraffin-embedded, sections $(5 \mu \mathrm{m})$ were prepared, and IHC was performed with monoclonal antibodies against $\alpha$-SMA and vimentin (Maxin). The IHC protocol was described as previously (24). H\&E staining and Masson trichrome staining (Maiwei) were performed on the three kinds of specimens. Images were obtained using laser confocal and optical microscopes.

Primary culture of normal fibroblasts (NFs) and CAFs. Fresh invasive cancer specimens and paired adjacent normal breast tissue samples ( $>3-5 \mathrm{~cm}$ away from the tumor) were collected under sterile conditions. The specimens were sectioned into approximately $1-\mathrm{mm}^{3}$ pieces and placed in DMEM/F12 (Gibco) supplemented with 10\% FBS (Gibco), antibiotics (100 U/ml penicillin and $100 \mu \mathrm{g} / \mathrm{ml}$ streptomycin; Sigma), ascorbic acid (20 ng/ml), and FGF-basic (10 ng/ml; Sigma). After one week of incubation in a humidified incubator with $5 \% \mathrm{CO}_{2}$ atmosphere, tissue debris was removed. Once primary cells reached $80 \%$ confluence, they were harvested and reseeded in a flask.

Characterization of CAFs/NFs. CAFs and NFs were seeded into 24-well plates. The culture medium was removed and cells were fixed for $20 \mathrm{~min}$ with SafeFix solution (Sinai) once $70-90 \%$ confluence was reached, followed by treating with $0.2 \%$ Triton $\mathrm{X}-100$ for $15 \mathrm{~min}$, and incubating in non-immunone goat serum for $20 \mathrm{~min}$. Rabbit anti-vimentin, anti-FSP-1, anti-fibronectin antibodies and mouse anti$\alpha$-SMA, anti-cytokeratin (pan) antibodies (Maxin) were used in incubation at $4^{\circ} \mathrm{C}$ as the primary antibody. Anti-mouse and anti-rabbit fluorescent secondary antibodies were then applied accordingly. After rinsing, cells were counterstained with DAPI (Sigma), and images were obtained with a laser scanning confocal microscope. Immunocytochemistry by DAB staining was conducted. The methods for western blot analysis are presented in the section 'Western blot analysis'.

Cell growth evaluation. Briefly, 1,500 CAFs and NFs were seeded per well in 96-well plates. Cell viability was assessed daily by adding $20 \mu \mathrm{l}$ of 3-(4,5-dimethylthiazol-2-thiazyl)2,5-diphenyl-tetrazolium bromide (MTT; $5 \mathrm{mg} / \mathrm{ml}$ in PBS) to each well. MTT was also added to the control wells without cells. Each sample included six replicates. Following 4-h incubation at $37^{\circ} \mathrm{C}$, the medium was removed. An aliquot of $200 \mu \mathrm{l}$ DMSO was transfered to each well, and the plate was agitated for $15 \mathrm{~min}$. The absorbance at $570 \mathrm{~nm}$ was then detected. For MTT assays that were performed on the same day with cell seeding, the cells were allowed to attach for $3 \mathrm{~h}$ before the addition of MTT. Growth curves were constructed by plotting absorbance (mean $\pm \mathrm{SD}$ ) against time.

Extraction of secretory proteins from CAFs and NFs. When CAFs and NFs reached $70-80 \%$ confluence, the medium was removed, and the flasks were washed gently three times with PBS. Cells were then cultured in conditioned medium (CM; serum-free, phenol red-free DMEM medium supplemented with ascorbic acid $20 \mathrm{ng} / \mathrm{ml}$ ). Following 18-h incubation, the $\mathrm{CM}$ was collected and the cell debris was removed by centrifugation for $10 \mathrm{~min}, 4^{\circ} \mathrm{C}(200 \mathrm{x} \mathrm{g})$, followed by sterile filtration (0.22 $\mu \mathrm{m}$; Millipore). Protease inhibitors were applied to prevent protein degradation. The protein present in the CM was concentrated by an ultrafiltration (3,000 Da; Millipore) and precipitated in acetone overnight at $-20^{\circ} \mathrm{C}$. The sediment was then washed twice with acetone, followed by resuspended in protein solution (RIPA, Beyotime). The protein concentration was then measured with a standard Bradford protein assay (Bio-Rad). Prior to the collection of condition medium, $\beta$-galactosidase staining was performed to exclude senescent cells. CM samples were collected from three pairs of homologous CAFs and NFs.

MALDI TOF/TOF-MS for global screening of secretory protein. In total, $200 \mu \mathrm{g}$ of secretory proteins from CAFs and NFs were separated by $12 \%$ SDS-PAGE, and the resulting gel was stained with Commassie Blue Fast Staining Solution 

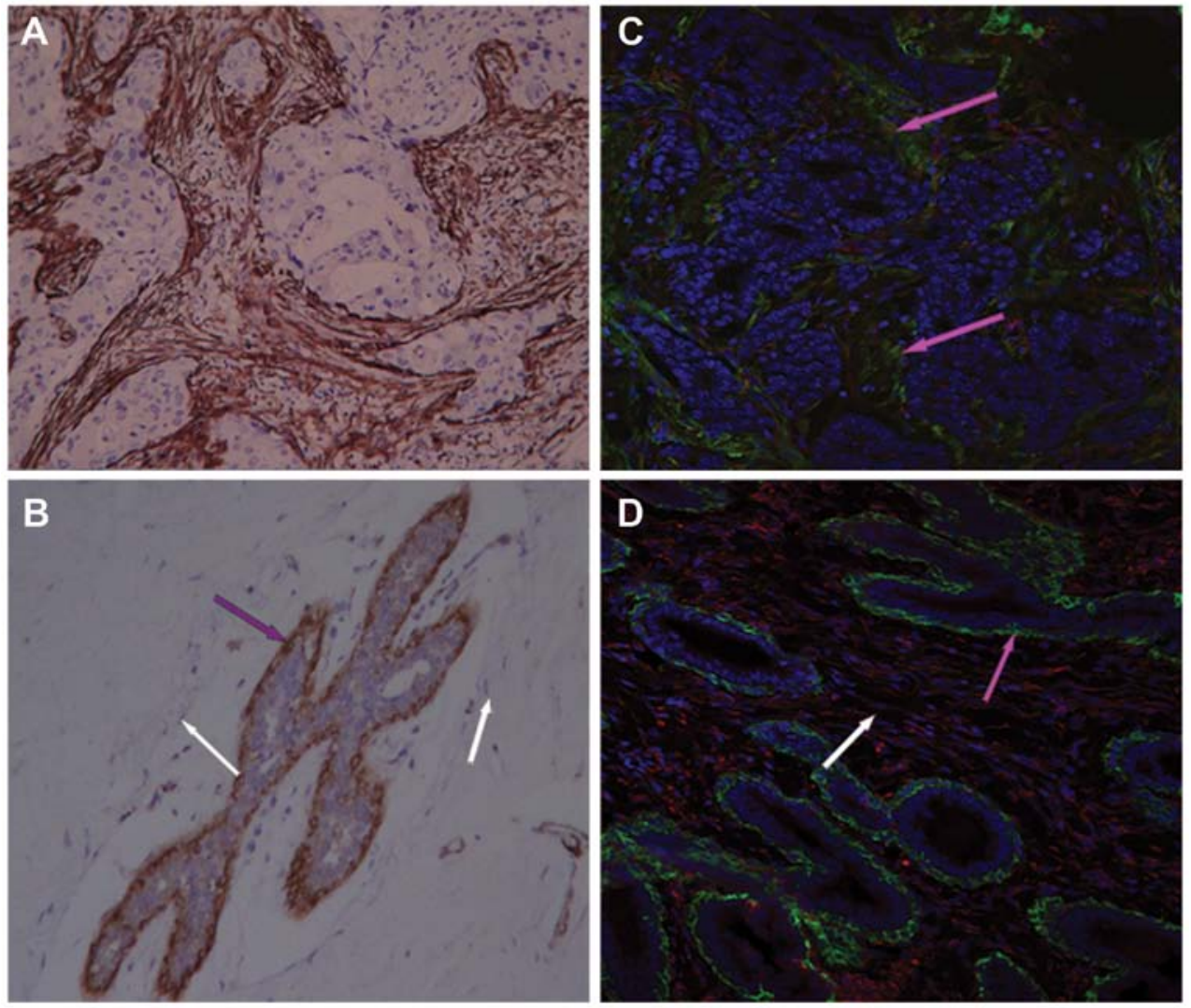

Figure 1. The difference of $\alpha$-SMA expression in invasive breast cancer, normal breast tissue and breast fibroadenoma. Tissues detected by immunohistochemistry and immunofluorescence showed that: $\alpha$-SMA expression was widely observed in the stroma of invasive breast cancer tissue [the brown area in (A), DAB staining. (C) Green, $\alpha$-SMA; red, vimentin, pink arrows]. While, little $\alpha$-SMA expression was observed in normal breast/fibroadenoma stromal region (B, white arrows), and $\alpha$-SMA was only present in myoepithelium, vimentin was negative (B and D, pink arrows, no red). (A, B, C and D), 200-fold.

(Invitrogen). Each lane was then cut into 15 even sections and digested as previously reported (25). Briefly, all sections were destained and dehydrated, and proteins were reduced with dithiothreitol and alkylated with iodoacetamide (IAA, Sigma). After alkylation, the samples were incubated with sequencing-grade trypsin (Promega) at $37^{\circ} \mathrm{C}$ for $20 \mathrm{~h}$. The peptides were then subjected to extraction $(50 \%$ acetonitrile, 5\% formic acid) and lyophilized under vacuum for MALDI TOF/TOF (Bruker Ultraflextreme) detection. All of the peptides were retrieved from the UniProtKB website.

Label-free quantitative assay for secretory proteins of interest Trypsin digestion. Protein samples from each fraction were reduced with DTT and alkylated with IAA. After being diluted in a solution of $100 \mathrm{mM} \mathrm{NH}_{4} \mathrm{HCO}_{3}$, the protein mixture was digested by sequencing-grade trypsin at $37^{\circ} \mathrm{C}$ for $20 \mathrm{~h}$.

Desalting peptides and multiple reaction monitoring (MRM) assay. The tryptic peptide mixture was desalted using a porous C-18 reversed-phase resin (Pierce) according to the manufacturer's instructions. Eluted peptides were lyophilized and redissolved in $10 \mu 10.1 \%$ FA for 4000 QTRAP $^{\circledR}$ LC/MS/MS System and targeted proteomics assay (MRM assay model) (26).

\section{Western blot analysis}

Protein extraction. Whole intracellular proteins were extracted in accordance with a standard protocol. Briefly, adherent cells (within 8 passages) were washed twice with PBS, and then lysed in RIPA and ultrasonicated on ice. The lysates were then centrifuged for $30 \mathrm{~min}$ at $4^{\circ} \mathrm{C}(10,000 \mathrm{x} \mathrm{g})$ and the supernatants were collected and stored at $-80^{\circ} \mathrm{C}$. The extraction of secretory proteins was performed as outlined in 'Extraction of secretory proteins form CAFs and NFs' section.

Western blots. Equal amounts of protein samples from CAFs and NFs (intracellular or secretory proteins) were separated on a $12 \%$ SDS-PAGE gel, transferred to PVDF membranes $(0.2 \mu \mathrm{m}$; Millipore), and then incubated with various primary antibodies ( $\alpha$-SMA, PDGFR- $\beta$, collagen $\alpha-\mathrm{I}$, collagen $\alpha$-III, diluted $1: 2,000$ in $5 \%$ defatted milk, GADPH, diluted $1: 5,000)$ overnight at $4^{\circ} \mathrm{C}$. The membranes were blotted with HRP conjugated secondary antibodies (Epitomics), developed using an ECL substrate (Pierce) and exposed to Kodak Biomax MR film.

Bioinformatics analysis and statistical analysis. MS data were analyzed using Analyst software (version 1.5.1, AB Sciex), and sequences were searched in the SwissProt database with Protein Pilot (version 4.0, AB Sciex). The cellular localization of identified proteins was analyzed on the basis of information available from UniprotKB. MRM propilot (version 2.1, AB Sciex) and Protein Pilot were used to analyze the amino acid sequence of trypsin-digested peptides and determine the optimal peptide sequences for quantitative detection. Statistical analyses were conducted with SPSS software (version 19.0). 


\section{Results}

Fibroblast activation in breast cancer. To characterize the activation of fibroblasts, the expression of $\alpha$-SMA was analyzed in invasive breast cancer, normal breast tissue and fibroadenoma. We found the $\alpha$-SMA positive fibroblasts were widely present in the stroma of invasive breast carcinoma compared to that of normal breast tissues and fibroadenoma (Fig. 1). In normal breast tissues, only the myoepithelial cells distributed along the entire duct-lobular system and the smooth muscle cells around the microvasculature were $\alpha$-SMA positive, while vimentin was not detected. It has been reported that fully differentiated myoepithelial cells can vanished during cancer progression (27). Therefore, the $\alpha$-SMA positive cells within the tumor stroma activated CAFs $\left(\alpha-\mathrm{SMA}^{+}\right.$vimentin $\left.{ }^{+}\right)$. A large number of fibroblasts were detected in breast fibroadenoma with abundant ECM, however, these fibroblasts were $\alpha$-SMAnegative. In summary, our results suggest that activation of fibroblasts only appeared in invasive cancer, which is consistent with findings from previous studies (13). Noteworthy, our data showed that not all the CAFs showed $\alpha$-SMA expression in the immunofluorescence assay.

CAFs maintain an activated phenotype in vitro. To investigate the characteristics of the primarily cultured cells, we performed immunocytochemistry and immunofluorescence using antibodies against $\alpha$-SMA, the muscle cell marker desmin, the epithelial cell marker cytokeratin (pan), the mesenchymal cell marker vimentin, the fibroblast marker FSP-1 and fibronectin (Fig. 2). The results indicated that only CAFs were $\alpha$-SMA-positive, while vimentin and fibronectin could be detected in both CAFs and NFs. Although FSP-1 was expressed in both cell types, relatively few positive cells were observed. Our results demonstrated that cytokeratin was not expressed in either type of cells. It was notable that the CAFs from one specimen showed weak expression of desmin which would be expected to be absent in breast fibroblasts. The expression of $\alpha$-SMA was confirmed by western blot analysis (Fig. 2I). Furthermore, another activation marker PDGFR- $\beta$ was detected by western blot analysis (Fig. 2I) but the result showed no difference between CAFs and NFs. These results demonstrated that CAFs and NFs were kindred cell types with the nature of fibroblasts after multiple passages in vitro, CAFs maintained an activated phenotype.

The proliferative capacity of CAFs and NFs. The MTT assays were carried out to analyze the proliferation capability of CAFs and NFs. The results were normalized statistically (Fig. 3A), suggesting that the proliferation ability of CAFs were either stronger than or equal to that of NFs (days 5, 6 and 7, p $<0.05$ or $\mathrm{p}>0.05$ ).

The detection of secretory proteins from CAFs and NFs. The 3 pairs of samples of secretory proteins obtained from $\mathrm{CM}$ of CAFs and NFs were globally scanned by MALDITOF/TOF-MS. Results showed in total 2,903 proteins in CAFs and 3,023 proteins in NFs (data not shown). Among them, 2,811 proteins were shared in these two samples. The extracellular proteins included matrix metalloproteinases (MMP1, MMP2, MMP3, MMP9, MMP10, MMP11 and MMP14), cathepsins, plas-
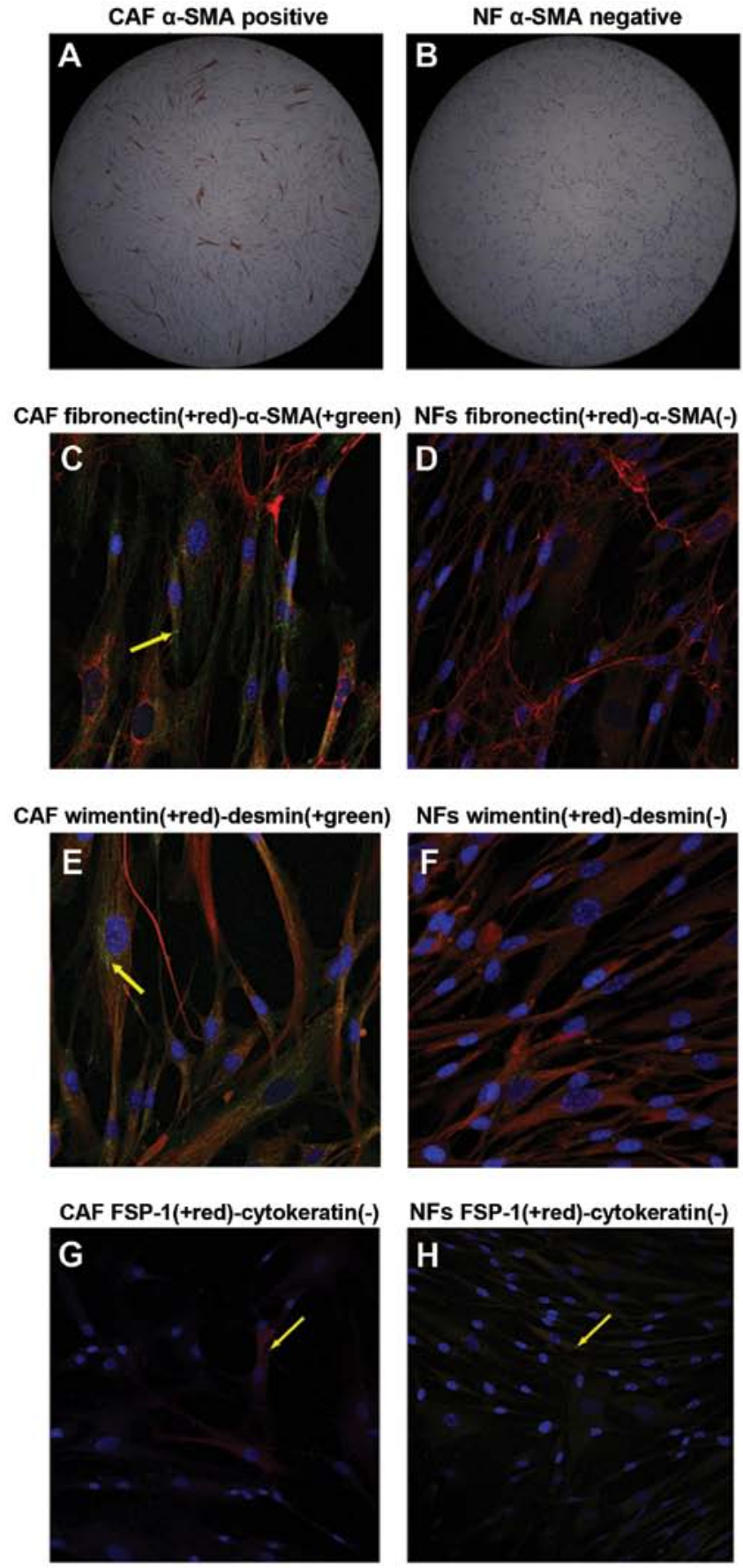

I Western blotting $\alpha$-SMA and PDGFR- $\beta$

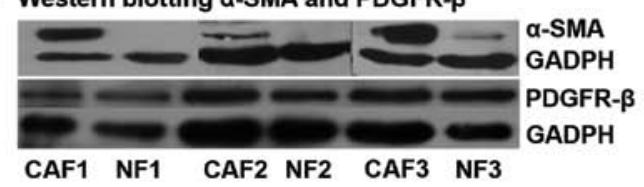

Figure 2. Characterization of CAFs and NFs activation. 1) Immunocytochemistry: The cells were labeled by DAB, fluorescent secondary antibodies (green, red) and DAPI (blue). a) Immunocytochemistry showed CAFs were fractionally $\alpha$-SMA-positive (A and C), while NF completely lacked $\alpha$-SMA expression (B and D). b) CAFs and NFs both express vimentin (E and F). In (E), CAFs demonstrated weak expression of desmin, which was absent in NF. c) No expression of cytokeratin (pan) was observed in CAFs or NFs, while the expression of FSP-1 was found only in a small proportion of CAFs and NFs (G and H). (A and B), 50-fold; (C, D, E, F, G and $\mathrm{H}$ ), 200-fold. 2) Western blot analysis for $\alpha$-SMA and PDGFR- $\beta$ assay: CAFs expressed $\alpha$-SMA, while NF almost completely lacked it. But, there was no expression difference on PDGFR- $\beta$ between CAFs and NFs (I). 

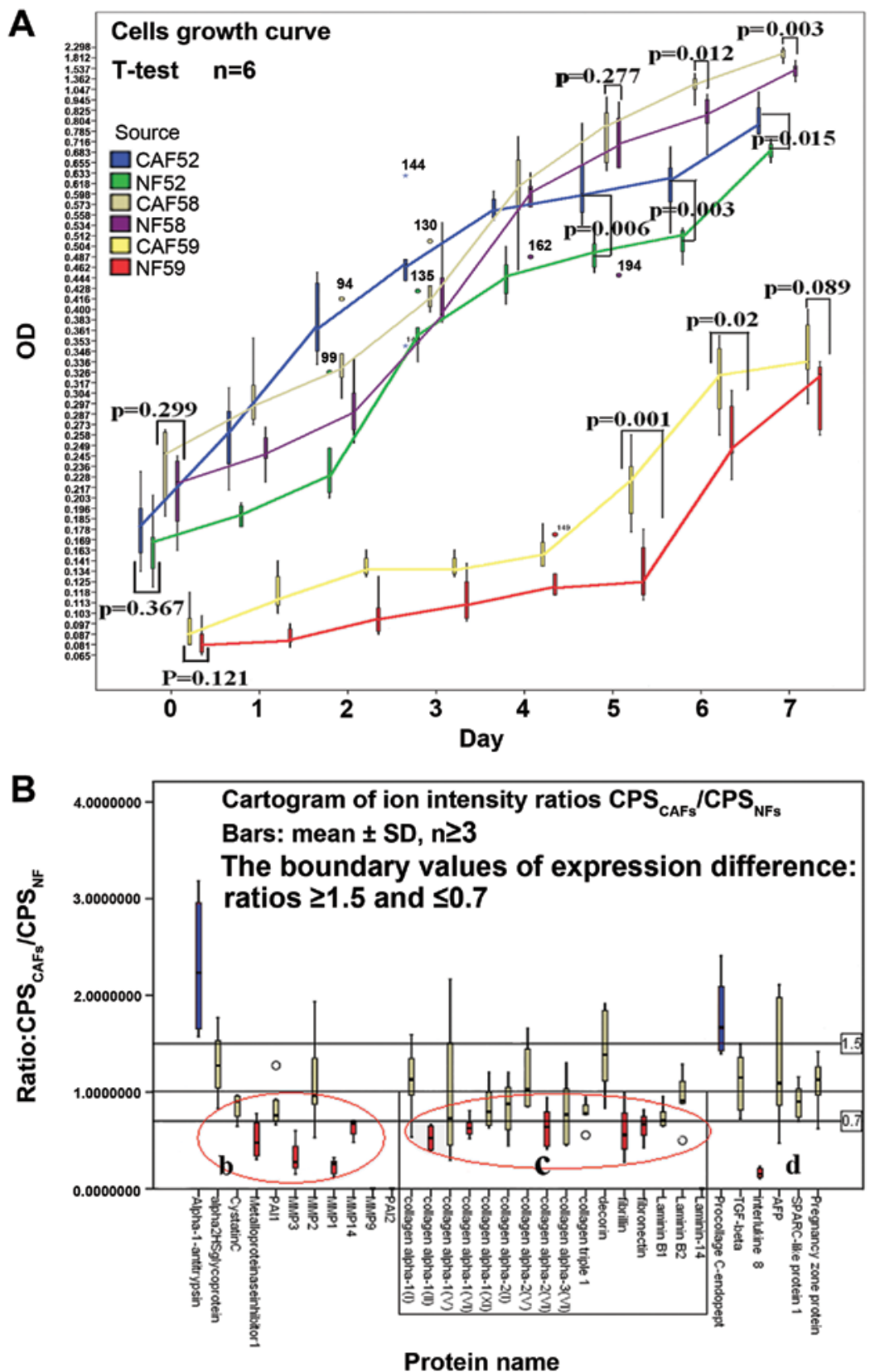

Figure 3. Cell growth curve and cartogram of secretory protein expression from CAFs and NFs. 1) Chart A shows the growth curve of CAFs and NFs. Three pairs of homologous CAFs and NFs were evaluated. On days 5,6 and 7, the data did not provide evidence that the proliferation of CAFs in vitro was weaker than that of NFs ( $<<0.05$ or $p>0.05$ ). 2) Chart B is a cartogram of peptide ion intensity ratios (CPSCAFs/ CPSNFs, mean \pm SD, $n \geq 3$ ). To delimit the expression difference, ratios $\geq 1.5$ and $\leq 0.7$ were specified as the difference boundary values of protein expression, and ratio $=1$ was assigned as an equivalent reference line. According to these rules, only two proteins in the selected proteins had higher expression in CAFs (blue), while more proteins showed lower expression (red). Chart B is divided into three parts: part b, matrix-degrading enzymes and protease inhibitors; part c, extracellular matrix components; and part d, other proteins. In most of the ratios parts b and $\mathrm{c}$ are lower than 1. These proteins included the EMC ingredient.

minogen activator inhibitors (PAI), cystatins, collagens and other extracellular components. Several membrane proteins were also identified, including cadherin, integrin and growth factor-binding protein. Regarding collagens, CAFs and NFs were found to have different expression profiles: collagen $\alpha-1(\mathrm{III})$, collagen $\alpha-5$ (VI) were only discovered in NFs, while collagen $\alpha-1(\mathrm{XII})$ and collagen $\alpha-2(\mathrm{~V})$ appeared in CAFs. Four of the six types were detected in CAFs.

Label-free quantitative assay for selected proteins by MRM. To quantitatively identify the differences between secretory proteins generated by CAFs and NFs, we chose to profile ECM-related proteins and other extracellular protein components, as well as several cytokines (Table I) by MRM, Applied Biosystems, and MDS Inc). The ion-intensity values (CPS) of representative peptides from different proteins were collected, and the CPS values of a same ion-peptide (in a same batch testing) were calculated, ion-intensity ratio $=\mathrm{CPS}_{\mathrm{CAFs}} / \mathrm{CPS}_{\mathrm{NFs}}$. A ratio of each protein was then obtained from data from the same batch testing. To delimit the expression difference, we specified ratios $\geq 1.5$ and $\leq 0.7$ as the boundaries of protein expression, with ratio $=1$ as an equivalent reference line. 
Table I. Selected extracellular proteins and peptides for quantitative assay by mass spectrometry (4000 QTrap LC/MS/MS, multiple-reaction monitoring model).

\begin{tabular}{|c|c|c|c|}
\hline Protein ID & Protein name & Abbreviation & The sequence of ideal peptides \\
\hline P02452 & Collagen alpha-1(I) chain & COL1A1 & $\begin{array}{l}\text { DGEAGAQGPPGPAGPAGER, ADDANVVR, } \\
\text { GPAGPQGPR }\end{array}$ \\
\hline P02461 & Collagen alpha-1(III) chain & COL3A1 & GPVGPSGPPGK \\
\hline P20908 & Collagen alpha-1(V) chain & COL5A1 & ENPGSWFSEEK \\
\hline P12109 & Collagen alpha-1(VI) chain & COL6A1 & $\begin{array}{l}\text { IALVITDGR, LKPYGALVDK, } \\
\text { TAEYDVAYGESHLFR, VPSYQALLR }\end{array}$ \\
\hline Q99715 & Collagen alpha-1(XII) chain & COL12A1 & $\begin{array}{l}\text { ALALGALQNIR, VILTPMTAGSR, } \\
\text { NSDVEIFAVGVK }\end{array}$ \\
\hline P08123 & Collagen alpha-2(I) chain & COL1A2 & $\begin{array}{l}\text { GEAGAAGPAGPAGPR, } \\
\text { TGEVGAVGPPGFAGEK, TGHPGTVGPAGIR }\end{array}$ \\
\hline P12110 & Collagen alpha-2(VI) chain & COL6A2 & DYDSLAQPGFFDR, LFAVAPNQNLK \\
\hline P12111 & Collagen alpha-3(VI) chain & COL6A3 & $\begin{array}{l}\text { QINVGNALEYVSR, QLGTVQQVISER, } \\
\text { VGLEHLR }\end{array}$ \\
\hline P05997 & Collagen alpha-2(V) chain & COL5A2 & SLSSQIETMR \\
\hline Q16363 & Laminin subunit alpha-14 & LAMA14 & AIEHAYQYGGTANSR \\
\hline P11047 & Laminin subunit gamma-1 & LAMC1 & $\begin{array}{l}\text { LSAEDLVLEGAGLR, } \\
\text { LVGGPMDASVEEEGVR, } \\
\text { NTIEETGNLAEQAR }\end{array}$ \\
\hline P07942 & Laminin subunit beta- 1 & LAMB1 & IPSWTGAGFVR \\
\hline P35555 & Fibrillin-1 & FBN1 & YLIESGNEDGFFK \\
\hline $\mathrm{P} 02751$ & Fibronectin & FN1 & $\begin{array}{l}\text { NLQPASEYTVSLVAIK, } \\
\text { NTFAEVTGLSPGVTYYFK, } \\
\text { TYHVGEQWQK, VGDTYERPK }\end{array}$ \\
\hline P14780 & Matrix metalloproteinase-9 & MMP9 & LGLGADVAQVTGALR \\
\hline P03956 & Interstitial collagenase & MMP1 & $\begin{array}{l}\text { SQNPVQPIGPQTPK, WEQTHLTYR, } \\
\text { VTGKPDAETLK, DGFFYFFHGTR }\end{array}$ \\
\hline Q14515 & SPARC-like protein 1 & SPARCL1 & LLAGDHPIDLLLR, MRDWLK \\
\hline P08254 & Stromelysin-1 & MMP3 & FLGLEVIGK \\
\hline P50281 & Matrix metalloproteinase-14 & MMP14 & SPQSLSAATAAMQK, AVDSEYPK \\
\hline Q96CG8 & Collagen triple helix repeat-containing protein 1 & CTHRC1 & ESFEESWTPNYK \\
\hline P20742 & Pregnancy zone protein & PZP & ATVLNYLPK, GPTQDFR \\
\hline P01033 & Metalloproteinase inhibitor 1 & TIMP1 & GFQALGDAADIR, SEEFLIAGK \\
\hline P01034 & Cystatin-C & CST3 & ALDFAVGEYNK, LVGGPMDASVEEEGVR \\
\hline P05121 & Plasminogen activator inhibitor 1 & PAI-1 & QVDFSEVER, TPFPDSSTHR \\
\hline P05120 & Plasminogen activator inhibitor 2 & PAI-2 & TPVQMMYLR \\
\hline Q15113 & Procollagen C-endopeptidase enhancer 1 & PCOLCE & GFLLWYSGR \\
\hline P07585 & Decorin & $\mathrm{DCN}$ & $\begin{array}{l}\text { VSPGAFTPLVK, AHENEITK, } \\
\text { DLPPDTTLLDLQNNK }\end{array}$ \\
\hline P01009 & Alpha-1-antitrypsin & SERPINA1 & QINDYVE \\
\hline P10145 & Interleukin 8 & & TYSKPFHPK \\
\hline P61812 & Transforming growth factor- $\beta$ & TGF- $\beta$ & $\begin{array}{l}\text { EGVYTVFAPTNEAFR, ILGDPEALR, } \\
\text { SPYQLVLQHSR }\end{array}$ \\
\hline P08253 & $72 \mathrm{kDa}$ type IV collagenase & MMP2 & $\begin{array}{l}\text { AFQVWSDVTPLR, } \\
\text { IIGYTPDLDPETVDDAFAR }\end{array}$ \\
\hline P02771 & Alpha-fetoprotein & AFP & YIQESQALAK \\
\hline
\end{tabular}

The expression differences of selected proteins are shown in Fig. 3B.

Our data showed $\alpha$-1-antitrypsin expression was upregulated, while metalloproteinase inhibitor 1 expression was downregulated in CAFs. No differences in protein expression were found for other proteinase inhibitors between CAFs and NFs. When metalloproteinases were tested, MMP1, MMP3 and MMP14 were found to be downregulated in CAFs compared with NFs. As for the ECM components, the data demonstrated that the expression levels of collagen $\alpha-1(\mathrm{II})$, collagen $\alpha-1(\mathrm{VI})$, 
A

a-1-antitrypsin

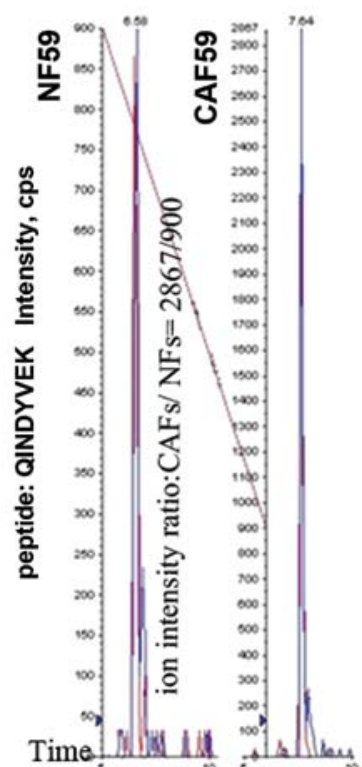

Collagen $\alpha-1(\mathrm{VI})$

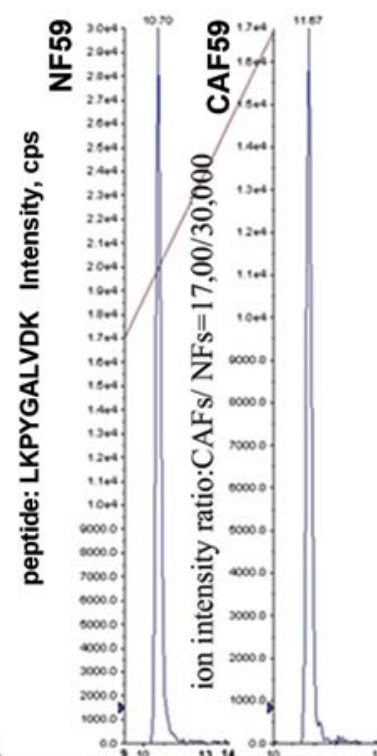

Stromelysin-1=MMP-3

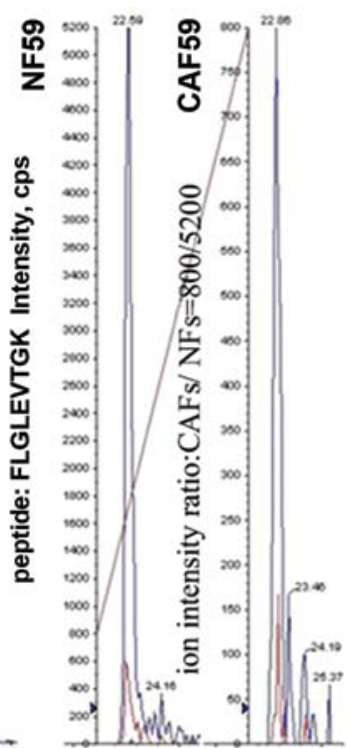

ion intensity difference of representative peptides from CÂFs and NFs
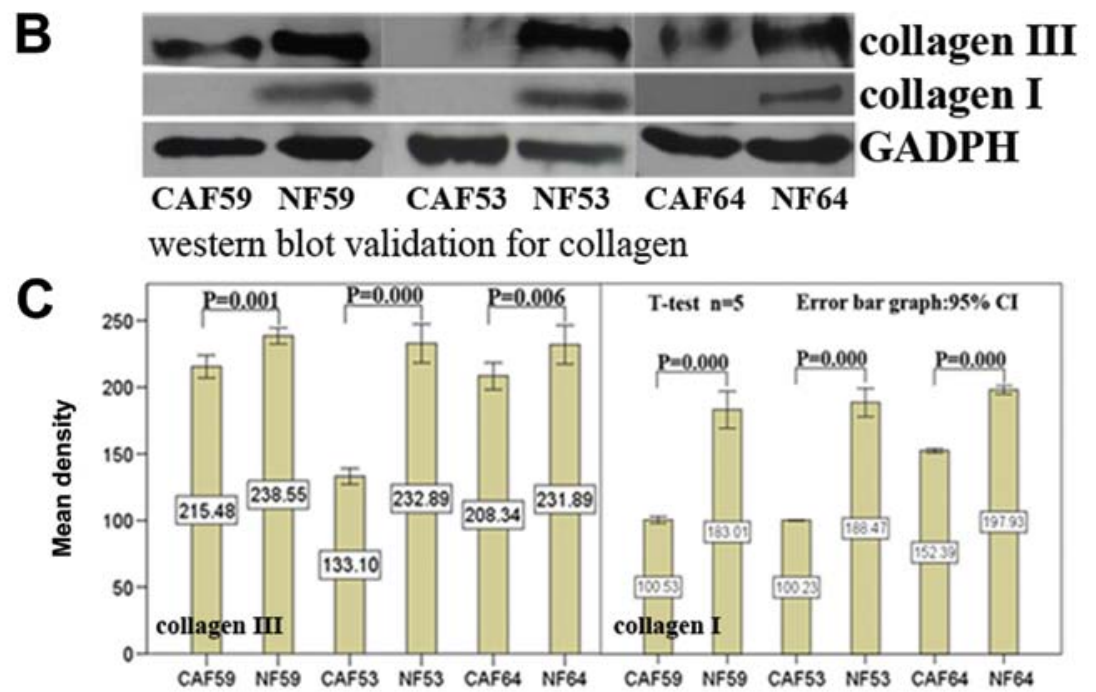

Figure 4. Validation of protein expression differences. (A) The ion intensity difference of representative paired peptides [ $\alpha-1$ antitrypsin, collagen $\alpha-1$ (VI) and MMP3] from homologous CAFs and NF. (B) The results of western blot analysis with anticollagen I and III antibodies (the bands appeared at approximate 95 and $190 \mathrm{kDa}$ ). Equivalent protein concentrations from paired homologous CAFs and NFs were loaded in every lane. GAPDH from paired CAFs, NFs was used as an internal control. (C) The grayscale statistical analysis of western blotting (collagen I and III; error bars, mean $\pm \mathrm{SD}, \mathrm{n}=3$ ).

collagen $\alpha-2(\mathrm{VI})$, fibrillin and fibronectin were all lower in CAFs than in NFs; only procollagen $\mathrm{C}$-endopeptidase enhancer 1 was highly expressed in CAFs. When ratio $=1$ was set as an equivalent reference line for the analysis, we found a broad decrease in expression of secretory proteins in CAFs.

Western blot analysis validation of collagen expression. In order to verify our results, we carried out western blotting to verify the collagen expression beyond the threshold $(\geq 1.5$ or $\leq 0.7)$. The results showed that there was a significant difference in expression of collagens between CAFs and NFs (Fig. 4). These results suggest that the setting of the boundary values was rational, and strengthened the reliability of data from our label-free quantitative assay. These findings also provide further evidence to prove the decreased expression of ECM components in CAFs.
Reduction of collagen deposition in breast cancer stroma. To further validate the collagen reduction found in the label-free quantitative assay, we performed $\mathrm{HE}$ and masson trichrome staining to assess the collagen density in breast cancer tissue, normal tissue and breast fibroadenoma. The HE staining sections suggested a significantly reduction of ECM amount in cancer stroma or cancer reactive stroma (Fig. 5A-C). Masson trichrome staining exhibited more obvious differences in collagen deposition. In order to quantify the results, the density of the blue color in masson trichrome staining was analyzed by ImagePro Plus6.0. Statistical analysis showed that there were significant differences in collagen deposition among cancer stroma, paracarcinoma, normal tissue and fibroadenoma, whereby cancer stroma showed the least collagen deposition $(\mathrm{p}=0.000)$ compared with the other three kinds of stroma. This assay and analysis further confirmed 


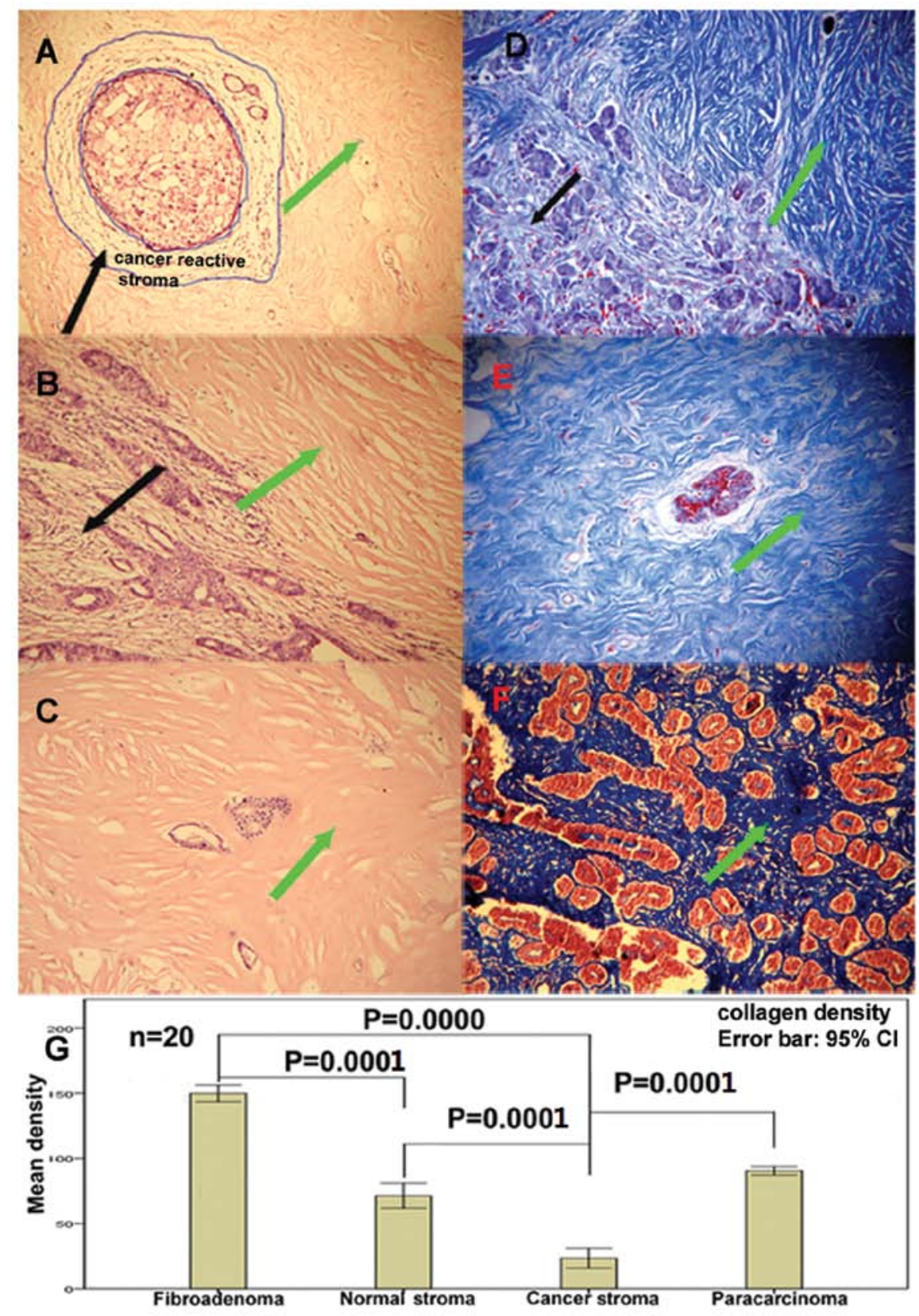

Figure 5. The deposition of ECM and collagen in tissue. 1) (A and B) H\&E staining showing that ECM is significantly reduced in cancer stroma or cancer reactive stroma, with or without increased numbers of stromal cells. (C) H\&E staining of normal breast tissue demonstrating a rich ECM and lack of stromal cells (green arrow). 2) (D, E and F) Masson trichrome staining depicting collagen in blue. In (D), the cancer stroma (black arrow) is lighter in blue color compared with paracarcinoma stroma, normal breast stroma (F) and fobroadenoma stroma (green arrow). The tissue of breast fibroadenoma showed the highest density of collagen deposition. 3) (G) Results of statistical analysis for collagen density (the blue area shown by masson trichrome staining. Four areas: cancer stroma, paracancer stroma, normal tissue stroma and fibroadenoma, in D, E and F). Cancer stroma showed the least collagen deposition comparing with the stroma from paracarcinoma, normal tissue and fibroadenoma (error bars represent mean \pm SEM, n=20). (A, B and C) 170-fold; (D, E and F) 130-fold.

the collagens reduction observed in the label-free quantitative assay. As collagens are the most abundant proteins of the ECM and offer structural support for resident cells, the decrease of collagen deposition implies a reduction of ECM in cancer stroma.

\section{Discussion}

Usually, the major function of fibroblasts is maintaining the structural framework of tissues by continuously secreting ECM components (28). Under normal conditions, fibroblasts stay inactivated by constitutively expressing vimentin and other markers (29). Once activated (by some pathological factors, for example, wound healing or fibrosis), they can immediately take on enhanced proliferative and ECM generating capability and become markers-positive, for example, $\alpha$-SMA and PGDFR- $\beta$. The series of responses finally leads to wound healing progression such as wound contraction, angiogenesis and stimulation of epithelial cell proliferation (30). It has been reported that CAFs in tumor microenvironments also acquire a similar activated phenotype, and function in a co-evolutionary manner with cancer cells $(31,32)$. 
In the present study, we determined the expression of $\alpha$-SMA, which is a major component of the contractile apparatus, as a marker of fibroblast activation. While there are other activation markers that can be used to reflect the fibroblast activation state, $\alpha$-SMA was chosen because it is the most commonly used. Immunohistochemistry demonstrated that CAFs were the only cellular components that expressed $\alpha$-SMA in the stroma of invasive breast cancer, but not in normal and benign tissues (Fig. 1). In agreement, Sappino et al have found that up to $80 \%$ of breast cancers include $\alpha$-SMA positive CAFs (33) while evidence from other studies has shown absence in breast fibroadenoma (34). Therefore, our data confirmed that the CAFs found in cancer stroma are in an activated state. We also found that after undergoing multiple passages (within 8 passages), primary cultured CAFs were still able to maintain the expression of activation marker $\alpha$-SMA (Fig. 2), suggesting that CAFs were able to maintain the activated state in vitro as well as in vivo.

Some researchers have indicated that activated CAFs with PDGFR- $\beta$ overexpression are responsible for the expansion of tumor stroma and the excessive deposition of ECM $(19,22)$. The increased deposition of ECM in cancer is called desmoplasia, which is a process similar to that in organ fibrosis. Usually, the desmoplastic stroma contains increased amounts of fibrillar collagens and other ECM components $(35,36)$. However, as ECM deposition in breast cancer tissues presents different pathological types (23), further study is required to disclose the true relationship between activated CAFs and ECM deposition. In this study, proliferation assays of CAFs and NFs did not provide evidence that the proliferation of CAFs in vitro was weaker than that of NFs. While our western blot analysis experiment showed no PDGFR- $\beta$ expression difference between CAFs and NFs. Based on the activation state of CAFs in vitro, we then examined secretory proteins from CAFs and NFs using MS. Many extracellular matrix components were detected, such as collagens, fibronectin, decorin and several proteases involved in ECM degradation (such as MMPs, cathepsin and PAI) were identified (data not shown). Besides the differences in the expression profile of secretory proteins between CAFs and NFs, the results of a labeled-free quantitative assay (MRM) for intresting proteins further demonstrated that the expression of ECM-related proteins and proteases from CAFs diminished compared to that of NFs; this was confirmed by western blot analysis (Fig. 4). Therefore, our experiments suggested that activated CAFs might be attenuated in their ability to produce ECM components, and biological characteristics of CAFs from invasive breast cancer are different from those of fibroblasts in normal breast tissue. This is in complete contradiction with the theoretical anticipation that activated CAFs would secrete a large amount of ECM components, more than NFs.

Tissue staining studies were carried out to further clarify the relationship between CAFs and ECM deposition in situ. In our study, the decline of ECM in malignant tissue was observed by HE staining. Comparing to that of paraneoplastic, normal breast and fibroadenoma stroma, Masson trichrome staining further demonstrated that the collagen within tumor stroma was significantly reduced. Additionally, fibroadenoma stroma was shown to have the most abundant collagen in our experiments.
While fibroblasts with activated traits are expected to produce more ECM components, evidence in our study demonstrated that normal breast stroma and fibroadenoma, rather than invasive breast cancer stroma had more extensive collagen deposition. The fibroblasts in normal breast stroma and fibroadenoma, however, are not $\alpha$-SMA-positive. The reduction of collagens within the tumor stroma might be possibly caused by an increase in the expression of matrix-degrading enzymes from tumor cells and tumor stromal fibroblasts, or by the decreased secretion of ECM components from CAFs. However, in this study, there was no evidence to suggest that there was an increase in the expression of MMPs and other proteases from CAFs (Fig. 3B). Our study therefore proposes that the reduction of collagen secretion in CAFs was the major cause of ECM reduction in tumor stroma.

In summary, although CAFs from invasive breast cancer obtained the activated phenotype, their capacity of producing ECM components was significantly impaired compared with normal fibroblasts and fibroadenoma fibroblasts. In breast cancer, CAFs might have remodeled the stromal structure and tumor microenvironment through changes in their biological characteristics and the profile of secretory proteins.

\section{Acknowledgements}

This study was supported by a grant from the National Natural Science Foundation of China (nos. 83172210 and 30973382). We thank Dr Shanwei Wang and Dr Jiaping Peng for their technical support in pathology. We also thank Dr Jiekai Yu, Dr Jiawei Zhang and Dr Weiting Ge for their help in Mass Spectrometry and Laser Confocal technology.

\section{References}

1. Zeisberg M, Strutz F and Muller GA: Role of fibroblast activation in inducing interstitial fibrosis. J Nephrol 13 (Suppl 3): S111-S120, 2000.

2. Darby IA and Hewitson TD: Fibroblast differentiation in wound healing and fibrosis. Int Rev Cytol 257: 143-179, 2007.

3. Ryan GB, Cliff WJ, Gabbiani G, Irle C, Statkov PR and Majno G: Myofibroblasts in an avascular fibrous tissue. Lab Invest 29: 197-206, 1973.

4. Ryan GB, Cliff WJ, Gabbiani G, et al: Myofibroblasts in human granulation tissue. Hum Pathol 5: 55-67, 1974.

5. Watts GT: Myofibroblasts. Lancet 1: 335, 1979.

6. Ratajska A and Gawlik Z: Morphological and physiological characteristics of myofibroblasts. Patol Pol 33: 1-19, 1982 (In Polish)

7. Lorusso $\mathrm{G}$ and Ruegg C: The tumor microenvironment and its contribution to tumor evolution toward metastasis. Histochem Cell Biol 130: 1091-1103, 2008.

8. Tyan SW, Kuo WH, Huang CK, et al: Breast cancer cells induce cancer-associated fibroblasts to secrete hepatocyte growth factor to enhance breast tumorigenesis. PLoS One 6: e15313, 2011.

9. Pietras K and Ostman A: Hallmarks of cancer: interactions with the tumor stroma. Exp Cell Res 316: 1324-1331, 2010.

10. Bissell MJ and Hines WC: Why don't we get more cancer? A proposed role of the microenvironment in restraining cancer progression. Nat Med 17: 320-329, 2011.

11. Dvorak HF: Tumors: wounds that do not heal. Similarities between tumor stroma generation and wound healing. N Engl J Med 315: 1650-1659, 1986.

12. Vaheri A, Enzerink A, Rasanen K and Salmenpera P: Nemosis, a novel way of fibroblast activation, in inflammation and cancer. Exp Cell Res 315: 1633-1638, 2009.

13. Rasanen $\mathrm{K}$ and Vaheri A: Activation of fibroblasts in cancer stroma. Exp Cell Res 316: 2713-2722, 2010. 
14. Gialeli C, Nikitovic D, Kletsas D, Theocharis AD, Tzanakakis GN and Karamanos NK: PDGF/PDGFR signaling and targeting in cancer growth and progression: Focus on tumor microenvironment and cancer-associated fibroblasts. Curr Pharm Des 20: 2843-2848, 2014.

15. Bedekovics J, Kiss A, Beke L, Karolyi K and Mehes G: Platelet derived growth factor receptor-beta (PDGFRbeta) expression is limited to activated stromal cells in the bone marrow and shows a strong correlation with the grade of myelofibrosis. Virchows Arch 463: 57-65, 2013.

16. Jacob M, Chang L and Pure E: Fibroblast activation protein in remodeling tissues. Curr Mol Med 12: 1220-1243, 2012.

17. Liao D, Luo Y, Markowitz D, Xiang R and Reisfeld RA: Cancer associated fibroblasts promote tumor growth and metastasis by modulating the tumor immune microenvironment in a $4 \mathrm{~T} 1$ murine breast cancer model. PLoS One 4: e7965, 2009.

18. Eyden B: The myofibroblast: an assessment of controversial issues and a definition useful in diagnosis and research. Ultrastruct Pathol 25: 39-50, 2001.

19. Conti JA, Kendall TJ, Bateman A, et al: The desmoplastic reaction surrounding hepatic colorectal adenocarcinoma metastases aids tumor growth and survival via alpha $a_{v}$ integrin ligation. Clin Cancer Res 14: 6405-6413, 2008.

20. Schedin P and Keely PJ: Mammary gland ECM remodeling stiffness, and mechanosignaling in normal development and tumor progression. Cold Spring Harb Perspect Biol 3: a003228, 2011.

21. Barsky SH and Gopalakrishna R: An experimental model for studying the desmoplastic response to tumor invasion. Cancer Lett 35: 271-279, 1987.

22. Pandol S, Edderkaoui M, Gukovsky I, Lugea A and Gukovskaya A: Desmoplasia of pancreatic ductal adenocarcinoma. Clin Gastroenterol Hepatol 7: S44-S47, 2009.

23. Walker RA: The complexities of breast cancer desmoplasia Breast Cancer Res 3: 143-145, 2001.

24. Katikireddy KR and O'Sullivan F: Immunohistochemical and immunofluorescence procedures for protein analysis. Methods Mol Biol 784: 155-167, 2011.

25. Lai TC, Chou HC, Chen YW, et al: Secretomic and proteomic analysis of potential breast cancer markers by two-dimensional differential gel electrophoresis. J Proteome Res 9: 1302-1322, 2010.
26. Bluemlein $\mathrm{K}$ and Ralser $\mathrm{M}$ : Monitoring protein expression in whole-cell extracts by targeted label- and standard-free LC-MS/MS. Nat Protoc 6: 859-869, 2011.

27. Gudjonsson T, Adriance MC, Sternlicht MD, Petersen OW and Bissell MJ: Myoepithelial cells: their origin and function in breast morphogenesis and neoplasia. J Mammary Gland Biol Neoplasia 10: 261-272, 2005.

28. Grinnell F: Fibroblasts, myofibroblasts, and wound contraction. J Cell Biol 124: 401-404, 1994

29. McAnulty RJ: Fibroblasts and myofibroblasts: their source, function and role in disease. Int J Biochem Cell Biol 39: 666-671, 2007.

30. Tettamanti G, Grimaldi A, Rinaldi L, et al: The multifunctional role of fibroblasts during wound healing in Hirudo medicinalis (Annelida, Hirudinea). Biol Cell 96: 443-455, 2004.

31. Giannoni E, Bianchini F, Masieri L, et al: Reciprocal activation of prostate cancer cells and cancer-associated fibroblasts stimulates epithelial-mesenchymal transition and cancer stemness. Cancer Res 70: 6945-6956, 2010

32. Brentnall TA, Lai LA, Coleman J, Bronner MP, Pan S and Chen R: Arousal of cancer-associated stroma: overexpression of palladin activates fibroblasts to promote tumor invasion. PLoS One 7: e30219, 2012.

33. Sappino AP, Skalli O, Jackson B, Schurch W and Gabbiani G: Smooth-muscle differentiation in stromal cells of malignant and non-malignant breast tissues. Int $\mathbf{J}$ Cancer 41: 707-712, 1988.

34. Heneghan HM, Martin ST, Casey M, Tobbia I, Benani F and Barry KM: A diagnostic dilemma in breast pathology - benign fibroadenoma with multinucleated stromal giant cells. Diagn Pathol 3: 33, 2008.

35. Shao ZM, Nguyen M and Barsky SH: Human breast carcinoma desmoplasia is PDGF initiated. Oncogene 19: 4337-4345, 2000.

36. Iacobuzio-Donahue CA, Argani P, Hempen PM, Jones J and Kern SE: The desmoplastic response to infiltrating breast carcinoma: gene expression at the site of primary invasion and implications for comparisons between tumor types. Cancer Res 62: 5351-5357, 2002. 